\title{
Lagrangian alternative to QCD string
}

\author{
Alisa Katanaeva* \\ Saint Petersburg State University \\ E-mail: alice.katanaevadgmail.com

\section{Sergey Afonin} \\ Saint Petersburg State University \\ E-mail: afoninehep.phys.spbu.ru
}

The spectrum of radially excited hadrons provides much information about the confinement forces in QCD. We propose a framework for constructing the effective field models which describe this spectrum. Within our scheme, the hadrons acquire masses due to interaction with a scalar field modeling the non-perturbative gluon vacuum. The effective potential for this field is periodic with an infinite number of non-equivalent vacua. The radially excited hadrons emerge as elementary excitations over different vacua. We construct explicit examples for such effective theories in the meson sector. The existence of a classical field configuration in each vacuum is also observed.

The XXI International Workshop High Energy Physics and Quantum Field Theory

June 23 - June 30, 2013

Saint Petersburg Area, Russia

\footnotetext{
* Speaker.
} 


\section{Introduction}

The talk given was mostly based on the article [四] covering, however, a wider range of subjects.

A large number of observed hadron resonances can be viewed as radial excitations of some lighter hadrons due to identical quantum numbers. The form of spectrum of radial excitations is determined by the confinement forces in Quantum Chromodynamics (QCD). Thus the study of radial excitations seems to be indispensable to the understanding of the confinement dynamics.

The quark confinement and the radial excitations arise naturally in the hadron string models where the string serves as an approximation for the gluon flux-tube with nearly massless quarks at the ends [ [2, []]. This relativistic approach is well accommodated for the description of light mesons representing ultrarelativistic systems. Here the string serves as an approximation for the gluon flux-tube with nearly massless quarks at the ends. The semiclassical quantization of a thin flux-tube typically gives a linearly rising spectrum for masses squared:

$$
m_{k}^{2}=a(k+b), \quad k=0,1,2, \ldots,
$$

where the slope $a$ is proportional to the string tension. Since the latter is governed by the gluodynamics, the slope $a$ is expected to be unaffected by the quantum numbers of meson resonances. The known spectrum of light mesons seems to agree with the behavior (1.1).

The following observations were crucial for us and laid the foundation of our models:

- The usual models based on the QCD string possess an apt basic feature: the linear Regge recurrence, $m_{J}^{2} \sim J$ ( $J$ denotes the hadron spin), emerges already on the classical level of rotating string. However, the radial modes appear after quantization;

- A careful analysis of existing data shows that a stronger statement than the recurrence (1.1) can be made: The light non-strange mesons cluster near certain equidistant values of energy squared with repeating structure of the clusters [四].

In this work we put forward a new dynamical approach to the description of the radial hadron excitations in which the spectrum of radially excited states is realized on the classical level. The approach is based on a realization of the assumption that the gluon vacuum has the periodic structure.

In some sense, an example of such approach is given by the five-dimensional soft-wall holographic model. We wish to construct models staying within the four-dimensional framework.

\section{Scalar theory with non-degenerate vacua}

Consider the following effective field theory for a real scalar field in four dimensions,

$$
\mathscr{L}=\frac{1}{2} \partial_{\mu} \varphi \partial^{\mu} \varphi+\frac{\mu^{4}}{2 \lambda}\left[\cos \left(\frac{\lambda}{\mu^{2}} \varphi^{2}-2 \pi b\right)-\cos (2 \pi b)\right],
$$

where $\mu$ has the dimension of mass and the parameters $\lambda$ and $b$ are dimensionless. The periodicity of the interaction term in (2.1) imposes a restriction on the value of parameter $b$ : the interval $0 \leq b<1$ covers all nontrivial cases. 
At $b=0$ the Lagrangian (2.1) resembles the sine-Gordon model [ []]. The model (2.1), however, is quite different since the nonlinear power of $\varphi$ in the cosine destroys the translational invariance (modulo a general factor) $\varphi \rightarrow \varphi+2 \pi k, k=0, \pm 1, \pm 2, \ldots$. The vacua are therefore non-equivalent, although they have equal minimal energy.

The potential in (2.1) is minimized on the constant field configurations

$$
\langle\varphi\rangle_{k}=\sqrt{\frac{2 \pi \mu^{2}}{\lambda}(k+b)}, \quad k=0,1,2, \ldots
$$

Considering small perturbations around the vacua $\varphi=\langle\varphi\rangle_{k}+\sigma(x)$ one can easily receive the spectrum of excitations which has the Regge-like form (1.1):

$$
M_{\sigma, k}^{2}=2 \lambda\langle\varphi\rangle_{k}^{2}=4 \pi \mu^{2}(k+b) .
$$

Thus the model has an infinite number of unconnected vacua with different excitation energies given by the relation (2.3).

It is worth mentioning that a simple semiclassical quantization of the gluon string with massless quarks confined by the linear potential leads to the relation [6]: $M_{k}^{2}=4 \pi \tau k+$ const, where $\tau$ denotes the string tension. The mass parameter $\mu^{2}$ has thus an analogy with the string tension.

The seeming similarity of the Lagrangian (2.1) with the sine-Gordon model poses a question, whether there exist soliton-like solutions in the two-dimensional case. In fact, the most close analogue to the sine-Gordon model is provided by the following modification of the Lagrangian (2.1),

$$
\widetilde{\mathscr{L}}=\frac{1}{2} \partial_{\mu} \varphi \partial^{\mu} \varphi+\frac{\mu^{6}}{\lambda^{2} \varphi^{2}}\left[\cos \left(\frac{\lambda}{\mu^{2}} \varphi^{2}\right)-1\right] .
$$

The minima of effective potentials in (2.4) and in (2.1) at $b=0$ coincide. The equation of motion for the field $\varphi$ in the Lagrangian (2.4) has a soliton-like solution, which in the static case takes the form

$$
\varphi= \pm 2 \sqrt{\frac{\mu^{2}}{\lambda} \arctan e^{2 \mu\left(x-x_{0}\right)}} .
$$

The expression in (2.5) represents nothing but the square root of the static one-soliton solution in the sine-Gordon model [5]. The solution (2.5), however, is not a genuine soliton. The solution (2.5) describes a usual kink, which connects two neighbouring vacua, and in four dimensions it becomes a domain wall. The same situation takes place in the model (2.1), where the kinks exist but cannot be found analytically.

\section{Abelian Higgs model with periodic potential}

Consider now a $U(1)$ gauge model of the Nielsen-Olesen type [ $[\mathbb{}]$ in which the Higgs potential is taken from the scalar model (2.1). The Lagrangian reads

$$
\mathscr{L}=D_{\mu} \varphi\left(D^{\mu} \varphi\right)^{*}+\frac{\mu^{4}}{2 \lambda}\left[\cos \left(\frac{2 \lambda}{\mu^{2}} \varphi \varphi^{*}-2 \pi b\right)-\cos (2 \pi b)\right]-\frac{1}{4} F_{\mu \nu} F^{\mu \nu},
$$

where

$$
\varphi=\frac{\varphi_{1}+i \varphi_{2}}{\sqrt{2}}, \quad D_{\mu} \varphi=\partial_{\mu} \varphi-i e A_{\mu} \varphi, \quad F_{\mu \nu}=\partial_{\mu} A_{\nu}-\partial_{v} A_{\mu}
$$


The scalar field $\varphi$ acquires non-zero vacuum expectation values (2.2). Pointing these values along the $\varphi_{1}$, we will have an infinite set of massive fields $\varphi_{1}$ and massless fields $\varphi_{2}$ which represent the Goldstone modes, one for each vacuum in the absence of the gauge field $A_{\mu}$. The Goldstone modes are transformed into the longitudinal degrees of freedom of the gauge field due to the Higgs mechanism. This can be easily demonstrated by the standard change of the field variables,

$$
V_{\mu}=A_{\mu}-\frac{\partial_{\mu} \eta(x)}{e\langle\varphi\rangle_{k}}, \quad \varphi=\frac{\langle\varphi\rangle_{k}+\sigma(x)}{2} \exp \left(\frac{i \eta(x)}{\langle\varphi\rangle_{k}}\right)
$$

In terms of the variables (3.3) the quadratic part of the Lagrangian (3.1) is cast into the canonical form:

$$
\mathscr{L}_{\sigma, V}^{(2)}=\frac{1}{2} \partial_{\mu} \sigma \partial^{\mu} \sigma-\frac{1}{2} M_{\sigma}^{2} \sigma^{2}-\frac{1}{4} F_{\mu \nu} F^{\mu \nu}+\frac{1}{2} M_{V}^{2} V_{\mu} V^{\mu}+\text { const },
$$

where $M_{\sigma}^{2}$ is given by (2.3) and the masses of gauge field over different vacua become

$$
M_{V, k}^{2}=e^{2}\langle\varphi\rangle_{k}^{2}, \quad k=0,1,2 \ldots
$$

The relations (2.3) and (3.5) demonstrate that the infinite towers of massive vector and scalar particles emerge.

Let us seek for the Nielsen-Olesen vortex solutions. The equations of motion for the Lagrangian (3.1) are:

$$
\begin{gathered}
D^{\mu} D_{\mu} \varphi=\mu^{2} \varphi \sin \left(\frac{2 \lambda}{\mu^{2}} \varphi \varphi^{*}-2 \pi b\right), \\
\partial^{v} F_{\mu v}=i e\left(\varphi^{*} \partial_{\mu} \varphi-\varphi \partial_{\mu} \varphi^{*}\right)+2 e^{2} A_{\mu} \varphi \varphi^{*} .
\end{gathered}
$$

We will consider the static case, with the gauge choice $A_{0}=0$. Following the paper [ $\left.\square\right]$ we look for a cylindrically symmetric solution, with axis along the $z$-direction. The corresponding ansatz is [ $[$ ]

$$
\vec{A}(\vec{r})=\frac{\vec{r} \times \vec{e}_{z}}{r} A(r), \quad \varphi(\vec{r})=\chi(r) e^{i n \theta},
$$

where $\vec{e}_{z}$ denotes a unit vector along the $z$-direction and $n$ is an integer. In addition, it is assumed that $A_{\theta}=A(r)$ and $A_{r}=A_{z}=0$. The flux is given by $\Phi(r)=2 \pi r A(r)$, so that the magnetic field is

$$
B(r)=\frac{\Phi^{\prime}(r)}{2 \pi r}=\frac{(r A(r))^{\prime}}{r}
$$

where the prime stays for the derivative with respect to $r$. Inserting this ansatz to the equations of motion we arrive at

$$
\begin{gathered}
\frac{\left(r \chi^{\prime}\right)^{\prime}}{r}-\left[\left(\frac{n}{r}-e A\right)^{2}+\mu^{2} \sin \left(\frac{2 \lambda}{\mu^{2}} \chi^{2}-2 \pi b\right)\right] \chi=0, \\
\left(\frac{(r A)^{\prime}}{r}\right)^{\prime}-2 \chi^{2}\left(A e^{2}-\frac{n e}{r}\right)=0 .
\end{gathered}
$$

The exact solution of this system cannot be obtained analytically. We are going to find a solution with asymptotic behavior on the spacial infinity of the type $\chi \simeq$ const. Treating $\chi$ as a constant we 
get from the equation (3.11), with $C$ a constant of integration and $K_{V}$ the modified Bessel function of the second kind,

$$
A_{k}(r)=\frac{n}{e r}+\frac{C}{e} K_{1}\left(e\langle\varphi\rangle_{k} r\right)=\frac{n}{e r}+\frac{C}{e} \sqrt{\frac{\pi}{2 e\langle\varphi\rangle_{k} r}} e^{-e\langle\varphi\rangle_{k} r}+\ldots
$$

Then the magnetic field is

$$
B_{k}=-C\langle\varphi\rangle_{k} r K_{0}\left(e\langle\varphi\rangle_{k} r\right)=-C \sqrt{\frac{\pi\langle\varphi\rangle_{k}}{2 e r}} e^{-e\langle\varphi\rangle_{k} r}+\ldots
$$

Here the dots mean the lower order terms at $r \rightarrow \infty$. Substituting the asymptotics (3.12) for $A_{k}(r)$ into the equation (3.10) we obtain the approximate solution $\chi_{k} \simeq\langle\varphi\rangle_{k} / \sqrt{2}$, which defines the characteristic length $\Lambda$ (meaning the penetration length of the magnetic field in the condensed matter physics), $\Lambda_{k}=\left(e \chi_{k}\right)^{-1}$.

In contrast to the Nielsen-Olesen theory $[\square]$, here one formally has an infinite number of characteristic lengths, however, the real physical penetration length is determined by the largest one corresponding to $k=0$.

The original Nielsen-Olesen's idea was to identify the vortex-line solution with the Nambu dual string. The quantization of the latter had to give rise to the excited hadron spectrum. We deal with a quite different situation: the spectrum of excitation emerges on the classical level and each state can be accompanied by a vortex. This approach avoids all notorious problems with quantization of hadron strings. The existence of vortices might be speculatively interpreted as a footprint of real QCD string. However, most likely these solutions are just artefacts of the model.

\section{Discussions}

From the phenomenological point of view, the model under consideration predicts a linear Regge-like spectrum for radially excited scalar and vector states. In the general situation the slopes are different in contrast to the hadron string models where the universal slope is determined by the gluodynamics.

The existing experimental data on the light non-strange mesons seems to agree with the approximate universality of slopes of radial meson trajectories within the experimental errors [ [8]. We can easily incorporate this universality by setting a condition $e^{2}=2 \lambda$.

However, this entails a complete degeneracy of the scalar and vector states. If we associate the scalar field $\varphi$ with the scalar isoscalar particles ( $f_{0}$-mesons) and the vector field with the vector isoscalar states ( $\omega$-mesons) this prediction is not far from the reality, see Fig. 1 .

On the other hand we can keep the universal slope but remove the degeneracy. Consider a model where the real scalar field interacts with the massive gauge field. That corresponds to adding the vector part

$$
\mathscr{L}_{v}=-\frac{1}{4} F_{\mu v} F^{\mu v}+\frac{1}{2}\left(m_{v}^{2}+e^{2} \varphi^{2}\right) A_{\mu} A^{\mu}
$$

to the Lagrangian of the scalar model.

The models discussed above should be viewed as effective models for QCD in the large- $N_{c}$ limit (because the number of resonances is infinite and the zero-width approximation is implied). 


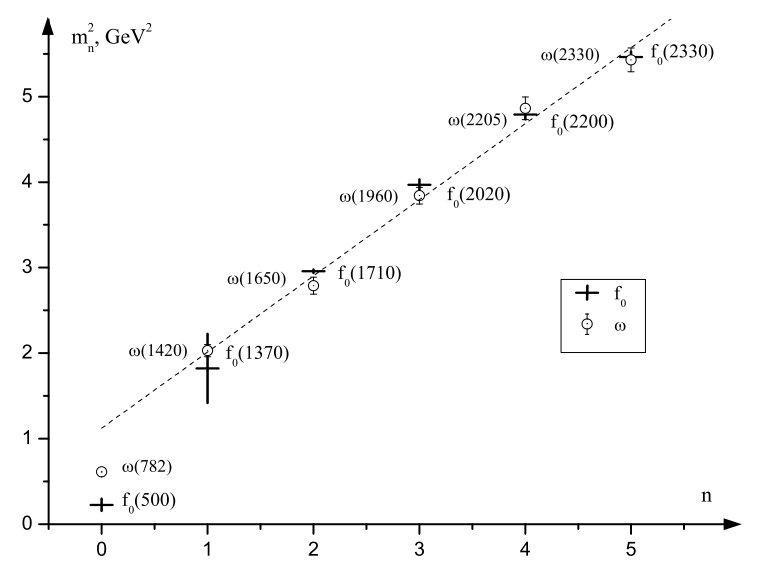

Figure 1: The spectrum of $f_{0}$ and $\omega$ mesons. The vertical size displays the experimental uncertainty. Other states $f_{0}$ reported by the Particle Data [Q] are assumed to belong to the $s \bar{s}$-trajectory. The state $\omega(2290)$ is identified with the $\omega(2330)$-meson.

They are complementary to the usual effective field theories for the strong interactions (the sigma-models, chiral perturbation theory, etc). The standard effective models are low-energy ones, i.e. they are usually defined below the characteristic scale of the spontaneous chiral symmetry breaking (CSB) - about $1 \mathrm{GeV}$ - and their input parameters are supposed to arise from integration of the degrees of freedom above this scale. In our case, the situation is opposite - by assumption, the input parameters (first of all the bare masses) originate from integration of the low-energy degrees of freedom below $1 \mathrm{GeV}$.

A phenomenological support for such a viewpoint comes from the observation that the linear ansatz (1.1) for the radial meson spectrum is a reasonable approximation for the excited states, while the ground ones below $1 \mathrm{GeV}$ deviate substantially from the linear trajectory. This is seen, for example, in Fig. I. The deviation is the most pronounced in the pseudoscalar channel [ $[$ ] $]$.

QCD in the large- $N_{c}$ limit represents a theory of an infinite number of narrow and stable non-interacting mesons and glueballs [ए]], which should appear in the classical effective action. It implies that we should introduce glueballs in our theory. In fact, we already have them for it looks rather natural to interpret the field $\varphi$ and its periodic potential as an effective model for the non-perturbative gluon vacuum in QCD.

It seems to be reasonable, that all quarkonia in the sector of light quarks get masses mainly through interaction with the vacuum field $\varphi$. Then the simplest Lagrangian for a scalar quarkonium $f$ is as follows from the equation

$$
\widetilde{\mathscr{L}_{s}}=\frac{1}{2} \partial_{\mu} f \partial^{\mu} f-\frac{1}{2}\left(m_{s}^{2}+g_{s}^{2} \varphi^{2}\right) f^{2}+\frac{1}{2} \partial_{\mu} \varphi \partial^{\mu} \varphi+\frac{\mu^{4}}{2 \lambda}\left[\cos \left(\frac{\lambda}{\mu^{2}} \varphi^{2}-2 \pi b\right)-\cos (2 \pi b)\right],
$$

From the Lagrangian (4.2) one can see that:

- the spectrum of the scalar glueballs has the Regge-like form;

- the bare mass $m_{s}$ must depend on quantum numbers in order to provide different intercepts for the radial meson trajectories [8];

- the approximate universality of slopes entails universality of couplings $g_{s}^{2} \simeq e^{2} \simeq 2 \lambda$. 
Finally we comment on the issue of renormalizability. The interaction in the Lagrangian (2.1) is non-renormalizable in four dimensions. Nevertheless, the effective field theories are often considered as approximations valid in the tree level only, and we imply no further accuracy.

\section{Conclusion}

In this work we proposed a new Lagrangian description for the radially excited mesons. The main assumption of our scheme is that the non-perturbative vacuum of QCD has periodic structure in energy scale.

We have built the models in which the Regge-like form of the spectrum of the radially excited states appears on the classical level. We have found that there is the Nielsen-Olesen vortex solution in each vacuum in the theory with Abelian gauge group. The existence of vortices might be speculatively interpreted as a footprint of real QCD string.

The case of non-Abelian gauge group as well as the calculation of the first quantum correction in the scalar model and some other subjects are discussed in the article [四].

\section{Acknowledgments}

The work was partially supported by the Saint Petersburg State University grants 11.38.660.2013 and 11.48.1447.2012, by the RFBR grant 13-02-00127-a and by the Dynasty Foundation.

\section{References}

[1] S. S. Afonin and A. D. Katanaeva, Eur. Phys. J. C 73, 2529 (2013).

[2] Y. Nambu, Phys. Rev. D 10, 4262 (1974); A. Casher, H. Neuberger and S. Nussinov, Phys. Rev. D 20 , 179 (1979); N. Isgur and J. E. Paton, Phys. Rev. D 31, 2910 (1985).

[3] D. LaCourse and M. G. Olsson, Phys. Rev. D 39, 2751 (1989); A. Yu. Dubin, A. B. Kaidalov and Yu. A. Simonov, Phys. Lett. B 323, 41 (1994); Yu. S. Kalashnikova, A. V. Nefediev and Yu. A. Simonov, Phys. Rev. D 64, 014037 (2001); T. J. Allen, C. Goebel, M. G. Olsson and S. Veseli Phys. Rev. D 64, 094011 (2001); M. Baker and R. Steinke, Phys. Rev. D 65, 094042 (2002); F. Buisseret, Phys. Rev. C 76, 025206 (2007).

[4] E. Klempt and A. Zaitsev, Phys. Rept. 454, 1 (2007); S. S. Afonin, Phys. Lett. B 639, 258 (2006); Eur. Phys. J. A 29, 327 (2006); Phys. Rev. C 76, 015202 (2007); Mod. Phys. Lett. A 22, 1359 (2007); M. Shifman and A. Vainshtein, Phys. Rev. D 77, 034002 (2008); L. Ya. Glozman, Phys. Rept. 444, 1 (2007).

[5] R. Rajaraman, Solitons and Instantons, North-Holland Publishing Company (Amsterdam, New York, Oxford, 1982).

[6] M. Shifman, hep-ph/0507246.

[7] H. B. Nielsen and P. Olesen, Nucl. Phys. B 61, 45 (1973).

[8] A. V. Anisovich, V. V. Anisovich and A. V. Sarantsev, Phys. Rev. D 62, 051502(R) (2000);

D. V. Bugg, Phys. Rept. 397, 257 (2004).

[9] J. Beringer et al. (Particle Data Group), Phys. Rev. D 86, 010001 (2012).

[10] G. 't Hooft, Nucl. Phys. B 72, 461 (1974); E. Witten, Nucl. Phys. B 160, 57 (1979). 